\title{
PATRONES BÁSICOS CAMINAR Y CORRER EN NIÑAS DE 5 A 8 AÑOS DE EDAD MEDIANTE EXTRACCIÓN NO LINEAL DE CARACTERÍSTICAS
}

\section{BASIC WALKING AND RUNNING PATTERNS IN 5 TO 8 YEAR OLD GIRLS BY NONLINEAR FEATURE EXTRACTION}

\author{
Santiago Ramos ${ }^{1}$, José A. Vidarte ${ }^{2}$, Luis A. Gómez ${ }^{3}$
}

\begin{abstract}
${ }^{1}$ Mag. Metodología Entren. Dep. ISCF Habana, Cuba. Grupo de Investigación Cumanday Actividad Física y Deporte, U. de Caldas, Manizales-Caldas, Colombia, e-mail: santiago.ramos@ucaldas.edu.co; ${ }^{2}$ Ph.D. en Ciencias. Act. Física y Deporte. Univ. Coruña, España. Grupo de investigación Cuerpo-movimiento, Univ. Autónoma de Manizales-Caldas, Colombia, e-mail: jovida@autonoma.edu.co; ${ }^{3}$ Ing. Electrónico Univ. Nacional de Colombia Manizales-Caldas, Colombia. Esp. Dirección y Gestión Deportiva Esc. Nal. Deporte, Cali-Valle del Cauca, Colombia. Dir. de Tecnología Sports Lab SAS, Manizales, Caldas, Colombia. Email: luisgt@laboratoriodeldeporte.com
\end{abstract}

Rev. U.D.C.A Act. \& Div. Cient. 17(2): 351-359, Julio-Diciembre, 2014

\section{RESUMEN}

Fueron caracterizados los patrones básicos de movimiento caminar y correr, en niñas de 5 a 8 años, residentes en áreas urbanas, mediante extracción no lineal de características. Se evaluaron 48 niñas, entre 5,7 y 8,9 años de edad (X 7,05 $\pm 0,99$ años), de un colegio privado de Manizales-Caldas, Colombia. Se emplearon técnicas de reconocimiento automatizado de patrones para obtener información acerca de posibles deficiencias motoras, fortalecer la detección temprana de falencias o deterioro biomecánico. La captura de imagen fue hecha con ocho cámaras de rayos infrarrojos que, mediante el programa OptiTrack Arena versión 1.7.0, permitieron formar esquemas de postura y posteriormente evaluados por expertos. Un consentimiento informado fue firmado por padres o acudientes. Las evaluadas, se desplazaron a velocidad submáxima autoelegida. Para propósitos de familiarización con la prueba, las participantes completaron dos intentos de práctica caminando o corriendo. Se encontró que un 57,14\% presentó anormalidades en el patrón de caminar y un $46,94 \%$, en el de correr. Frente a lo esperado para la edad, se concluyó que un $56,12 \%$ de las niñas evaluadas presenta patrones inmaduros para su edad, por lo que se recomienda incluir estos temas, con las respectivas correcciones, en los procesos de desarrollo motor.

Palabras clave: Desarrollo motor, marcha, desórdenes de la marcha, carrera, movimiento.

\section{SUMMARY}

Basic movement patterns, walking and jogging, were characterized in girls 5 to 8 years old living in urban areas, using non-linear features extraction. 48 girls were evaluated, aged between 5.7 to 8.9 years (mean $7.05 \pm 0.99$ years) from a private school on Manizales, Caldas, Colombia. Automated pattern recognition techniques were used to obtain information about possible motor deficiencies, strengthen early detection of flaws or biomechanical deterioration. Image capture was done with eight infrared cameras allowing through Arena OptiTrack version 1.7.0 form posture schemes, later assessed by experts. Informed consent was signed by parents or guardians. The evaluated individuals moved at submaximal auto elected speed. For the purpose of familiarization with the test, the participants completed two attempts to practice walking or running. It was found that a $57.14 \%$ had abnormalities in the walking pattern and a $46.94 \%$ in the running one. Faced with the expectancy for age, it was concluded that a $56.12 \%$ of the evaluated girls showed immature patterns for their age; therefore it is recommended to include these issues with the respective corrections in motor development processes.

Key words: Motor development, gait, gait disorders, running, movement. 


\section{INTRODUCCIÓN}

El crecimiento y el desarrollo se manifiestan, tanto en la estructura física como en el rendimiento motor e interactúan y se determinan genéticamente y desde lo social, cultural y económico (Barabas, s.f.). Los cambios en el desarrollo motor son entendidos como estados sucesivos de la estabilidad, que proporcionan el cambio de comportamiento motor de un estado a otro, posibilitando un mejor desempeño motor (Thelen \& Smith, 1994).

Anglada (2010) plantea que el ser humano nace con la necesidad de desplazarse, de cambiar de posición, porque esto contribuye, igualmente, a aumentar sus posibilidades de supervivencia. Los Patrones Básicos de Movimiento (PBM) son movimientos organizados espacio temporalmente (Muñoz, 1990), que forman la base para la adquisición y la realización de movimientos complejos y especializados, sobre los que se estructuran los diferentes deportes, caracterizados, principalmente, porque no se nace con pleno dominio sobre ellos (Silva, 2002).

La fase de los movimientos fundamentales ocurre durante los años del preescolar, que van desde los 2-3 años hasta los 6-7 (Frost, 2006; Mc Clenaghan \& Gallahue, 1985). Ellos están involucrados en el desarrollo y en el afinamiento de los PBM, tales como correr, saltar, lanzar y atrapar, que involucran movimientos finos y gruesos. Los niños en estas edades, primero aprenden las habilidades aisladas una de otra y, luego, son capaces de combinarlas, como movimientos coordinados (Frost et al. 2010; Hewes, 2006). Asimismo, Clark (2007) plantea que en la etapa pre-escolar y primaria temprana, se producen cambios y adaptaciones mucho más enmarcados en el sistema motor humano.

Caminar ha sido definido como una forma natural de locomoción vertical, caracterizada por la colocación alternada de pies, manteniendo contacto con el piso. El ciclo completo consiste en una fase de apoyo simple y otra de apoyo doble (Wickstrom, 1990). Se alternan las fases de balanceo con la de apoyo, siendo importante para el mantenimiento del equilibrio, que tiende a desaparecer, cuando la velocidad de locomoción aumenta y así la forma de locomoción pasa del andar al correr (Muñoz, 1990). Generalmente, se manifiesta al alcanzar el niño el primer año de edad, mostrando, normalmente, un paso corto, todavía muy vacilante, con los brazos ligeramente levantados, siempre buscando mantener el equilibrio (Meinel, 1977), conocida como posición de "guardia alta", descrita por Burnett \& Johnson (1971).

La secuencia propuesta por Seefeldt et al. (1972), para el patrón de la marcha, responde a cuatro estadios, donde cada uno de ellos establece unas características de desarrollo y de adquisición del patrón, hasta alcanzar su completa maduración.
A pesar que caminar es una forma aparentemente simple de desplazarse y que a los 3 años se pueden observar los patrones maduros de los ángulos articulares y coordinación brazos-piernas (Wickstrom, 1990), su forma madura a veces no se alcanza hasta la adolescencia, dependiendo del rigor que se utilice para evaluar su progreso.

El balanceo recíproco de los brazos sirve para incrementar el control postural del cuerpo y la eficiencia de la marcha; se contrabalancea la rotación del tronco alrededor del eje vertical (Ledebt, 2000) y se minimiza el desplazamiento vertical del centro de gravedad (Murray et al. 1967). El balanceo de los brazos no es una consecuencia pasiva del movimiento anterior del cuerpo, sino resultado de acciones musculares específicas en el hombro; no se observa en infantes al inicio de la marcha, pero se desarrolla durante la infancia (Burnett \& Johnson, 1971; Sutherland et al. 1980).

El patrón básico de correr es una forma más enérgica de locomoción, que permite alcanzar mayores velocidades con menor gasto de energía. Se define, como el conjunto de movimientos cíclicos, en los cuales, dos zancadas consecutivas realizan un ciclo completo de movimiento, en los que, a diferencia de caminar, se produce una fase de vuelo. Según Ruiz Pérez (1987), a partir de los 5 años, se puede apreciar el patrón maduro de la carrera, llegando a ser la forma predominante de movimiento a partir de los 6 años, en sus juegos espontáneos, aproximada ya mucho, en lo morfológico y biomecánico, a la del adulto (Meinel, 1977).

Para el análisis de la forma de correr, (Wickstrom, 1990) recomienda preguntarse si la pierna de apoyo está estirada al despegar, si se acerca el talón de la pierna libre al glúteo en la fase de recuperación, si se eleva bien la rodilla adelante, si se apoya el pie plano y debajo del cuerpo, si está el tronco vertical y la cabeza alineada y si se balancean ligeramente los brazos hacia la línea media con los codos flexionados.

Los PBM deben mejorar con la edad, ya que el desarrollo motor está sujeto a un proceso de maduración, mecanismo biológico relacionado con la edad cronológica, que conduce a la mejora de todas las funciones orgánicas (Gallahue \& Ozmun, 2005; Haywood, 1986), enfatizando en afirmar que el dominio PBM también depende del azar y la calidad de la práctica de las tareas abordadas (Clark, 2007; Gallahue \& Ozmun, 2005; Garcia et al. 2002; Goodway, 2009).

Como lo mencionan Ferraz (1992) y Braga et al. (2009), la falta de un adecuado desarrollo de los PBM fundamentales, se puede convertir en razón suficiente para que los niños no alcancen niveles de rendimiento motor adecuados y acordes a la edad cronológica, situación que pudiera afectar la participación eficaz en el juego, los bailes, el ocio y las actividades 
recreativas y las básicas cotidianas (Clark, 2007; Gallahue \& Donnelly, 2008).

La evaluación de los PBM busca establecer las deficiencias en su maduración para implementar, oportunamente, los correctivos, que garanticen la solución de deficiencias y un buen desempeño motor.

Ulrich (2000) aplicó la prueba de desarrollo motor grueso (Test of Gross Motor Development -TGMD-), a 1.208 niños de ambos sexos, de los 3 a los 10 años de edad, residentes en cuatro áreas geográficas de Estados Unidos. La gran mayoría de los niños presentaron un desempeño motor medio y, porcentajes más bajos, fueron encontrados en los extremos.

Algunos estudios muestran cómo el porcentaje de infantes, clasificados en las categorías de desempeño motor, desde las habilidades de locomoción, ha sido superior $0,6 \%$, encima de la media $0,7 \%$, media $25,5 \%$, debajo de la media $16,6 \%$, pobre $46,6 \%$ y muy pobre $9,9 \%$ (Braga et al. 2009; Gil-Agudo et al. 2009); esta clasificación los ubica en grupo $1(22,8 \%)$, grupo $2(42,9 \%)$ y grupo $3(33,3 \%)$ y, en las habilidades de manipulación, los valores fueron inferiores: grupo $1(71,4 \%)$, grupo $2(23,8 \%)$ y grupo $3(4,8 \%)$.

Dada la revisión presentada, el estudio se planteó como pregunta problema: ¿Es posible caracterizar los PBM caminar y correr en niñas de 5 a 8 años, mediante técnicas de extracción no lineal de características, que permitan una evaluación automática de dichos movimientos?

Como objetivo general, se buscó caracterizar los patrones básicos de movimiento: caminar y correr, en niñas de 5 a 8 años, residentes en áreas urbanas, por medio de técnicas de extracción no lineal de características.

\section{MATERIALES Y MÉTODOS}

Fue realizado un estudio cuantitativo, con alcance descriptivo, de corte transversal. La población objeto fueron niñas escolares de la ciudad de Manizales, Colombia, entre 5 y 8 años de edad, aparentemente sanas, de los grados transición, $1^{\circ}$ y $2^{\circ}$, de un colegio privado de la ciudad. La muestra, integrada por 48 niñas fue seleccionada al azar simple.

Los movimientos fueron registrados en 3D, empleando ocho cámaras de alta velocidad y luz infrarroja, reflejada en 34 marcadores reflectantes. Cada niña realizó, por lo menos, dos intentos exitosos de caminar y de correr dentro de un espacio de captura de movimiento, de 6 × 2 metros bajo techo.

Antes de cada bloque de ensayos, a las participantes se les dieron las siguientes instrucciones: "Inicie desde el cono de salida y camine (o corra, en el segundo patrón), hasta el cono de meta", "Camine (o corra) a una velocidad normal o cómoda".

Las imágenes fueron procesadas con el programa OptiTrack Arena, para ser convertidas en esquemas de postura y luego evaluados por dos expertos, como normales o anormales, anotándose, además, las discordancias entre las características de un patrón maduro, para su edad y las exhibidas por las escolares. Para éste propósito, se emplearon las recomendaciones de Mc Clenaghan \& Gallahue (1985), para la observación y evaluación de los PBM.

El tratamiento estadístico realizado fue análisis de frecuencias, en una base de datos levantada en el programa Excel bajo Windows. Los registros de video fueron adquiridos en condiciones semi-controladas de iluminación, con cámaras diseñadas para aplicaciones de captura de movimiento, a una velocidad de 100 cuadros por segundo y a una resolución 640 x 480 pixeles.

Sistema de captura de movimiento: Los equipos utilizados para la captura de movimiento pertenecen a los sistemas con sensores de tipo óptico, que identifican marcadores pasivos; se emplearon ocho cámaras OptiTrack V100:R2; tamaño de pixel: $6 \mu \mathrm{m} . \times 6 \mu \mathrm{m}$. latencia: $10 \mathrm{~m}$, resolución de imagen: $640 \times 480$, tipo de disparador: Global, frecuencia de muestreo configurable en: 25, 50, 100 FPS, velocidad de disparo: $1000 \mu$ s por defecto; mínima de $20 \mu$ s., precisión de sub-milímetro, anillo removible de 26 LEDs infra-rojos de $850 \mathrm{~nm}$., con brillo ajustable e iluminación configurable, como continua o estroboscópica.

Las señales de video en escala de grises fueron transportadas mediante cables USB hasta su concentrador; mediante el software, se pudo seleccionar cada una de las cámaras para ser vista individualmente, en niveles de grises.

Se emplearon dos concentradores OptiHub2 (USB2.0: High-Speed 480Mbps, Full-Speed (12Mbps), Low-Speed (1.5Mbps); se utilizó el esquema de conexión de los componentes del sistema, dado por el fabricante, colocando 8 cámaras y 2 concentradores USB.

Fueron utilizados marcadores reflectantes OptiTrack, fijadas a una base de velcro, con un tornillo de plástico. Fueron construidos trajes de captura de movimiento para los niños, de una sola pieza, dado que en el mercado no hay de tallas pequeñas, como se aprecia en la figura 1 . Todas las cámaras, se ubicaron a una altura de $1.90 \mathrm{~m}$, alrededor del volumen de captura, de modo que los marcadores reflectantes estuvieran a la vista de, por lo menos, dos cámaras simultáneamente. 


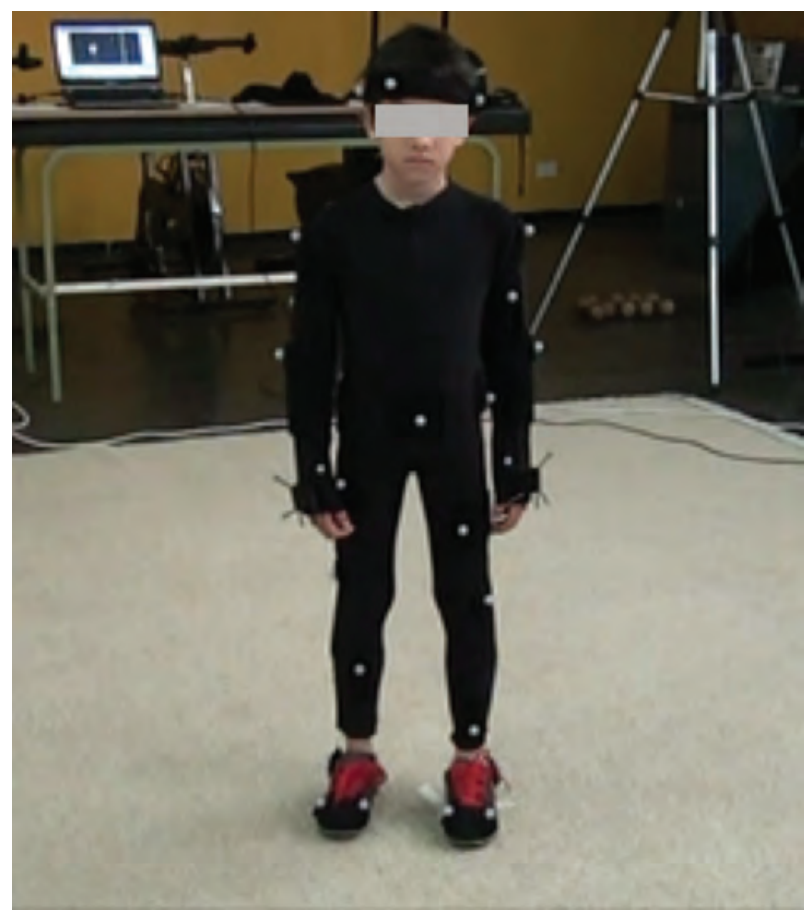

Figura 1. Niño vestido con el traje en acetato y los velcros para fijar los marcadores reflectantes.

Una vez realizada la calibración, se pudo observar la posición de cada cámara y la nube de puntos capturados en el proceso de calibración en el volumen 3D, en realidad virtual. De esta manera, se pudo comprobar el resultado del proceso de calibración, para determinar la captura de movimiento.

Los 34 marcadores ubicados en puntos específicos, para formar triángulos, para representar cada uno de los 16 segmentos corporales del modelo en 3D. Si los marcadores reflectantes fueron bien colocados, el software automáticamente asignaba los puntos del sujeto en posición "T" al modelo 3D, al ingresarle, como parámetros, la estatura y el diámetro biacromial, como se muestra en la figura 2.

Cada sujeto realizó los PBM caminar y correr, mientras se registraron secuencias de $30 \mathrm{~s}$, en las que se pre-visualizó que el gesto fuera realizado aceptablemente; cada secuencia, se guardó con un nombre, fecha y hora de la captura, el identificador del sujeto y del movimiento que se registró.

Los datos 2D capturados por las cámaras fueron procesados mediante técnicas de rastreo de múltiples puntos en movimiento; para generar un archivo con información 3D, filtrados con un filtro pasa-bajo de $6 \mathrm{~Hz}$, para suavizar las curvas y eliminar ruido que se hubiera introducido en la captura. Finalmente, los datos fueron exportados en el formato Biovision Hierarchy (BVH) (Figura 3). Este tipo de archivos fue procesado por el software implementado en Matlab, para realizar el apoyo en el análisis de los movimientos, por parte de los expertos en biomecánica y en educación física.

A partir del archivo $B V H$ obtenido por el sistema de captura 3D, se obtuvieron las características de importancia, según los expertos, para caracterizar y facilitar el análisis de la marcha y la carrera (Baena et al. 2010). La información personal y antropométrica básica del evaluado, siguió los protocolos de la ISAK (Ramos et al. 2007).

Aspectos éticos de registro: Los adultos responsables de las escolares fueron informados acerca del alcance del proyecto. Se solicitó su autorización para el uso de los registros, con el único fin de desarrollar la investigación, garantizando la reserva de sus identidades y con el derecho a retirarse del estudio, sin perjuicio alguno para la niña ni su tutor responsable. Estas condiciones fueron presentadas en un formato de consentimiento informado, avalado por el Comité de Bioética de la Facultad de Ciencias para la Salud, de la Universidad de Caldas.

Caracterización de los PBM: Los PBM fueron especificados a partir del estudio y revisión de literatura especializada para caminar y correr. La literatura del análisis biomecánico (Grosser et al. 1991; Kwon, 2009; Nitsch et al. 2002; Scheirman \& Hatfield, 2009) y del aprendizaje motor (Mc Clenaghan \& 


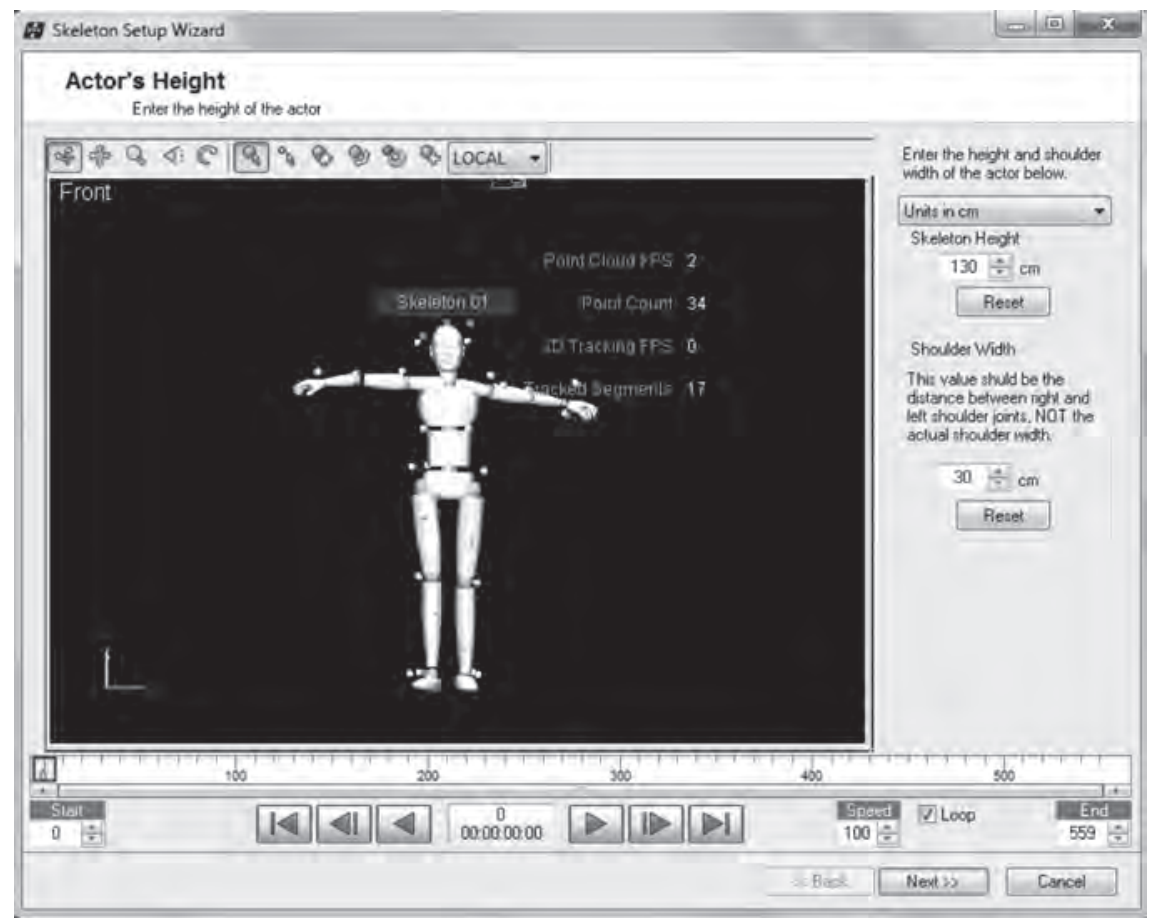

Figura 2. Configuración del esqueleto a partir de los marcadores reflectantes.
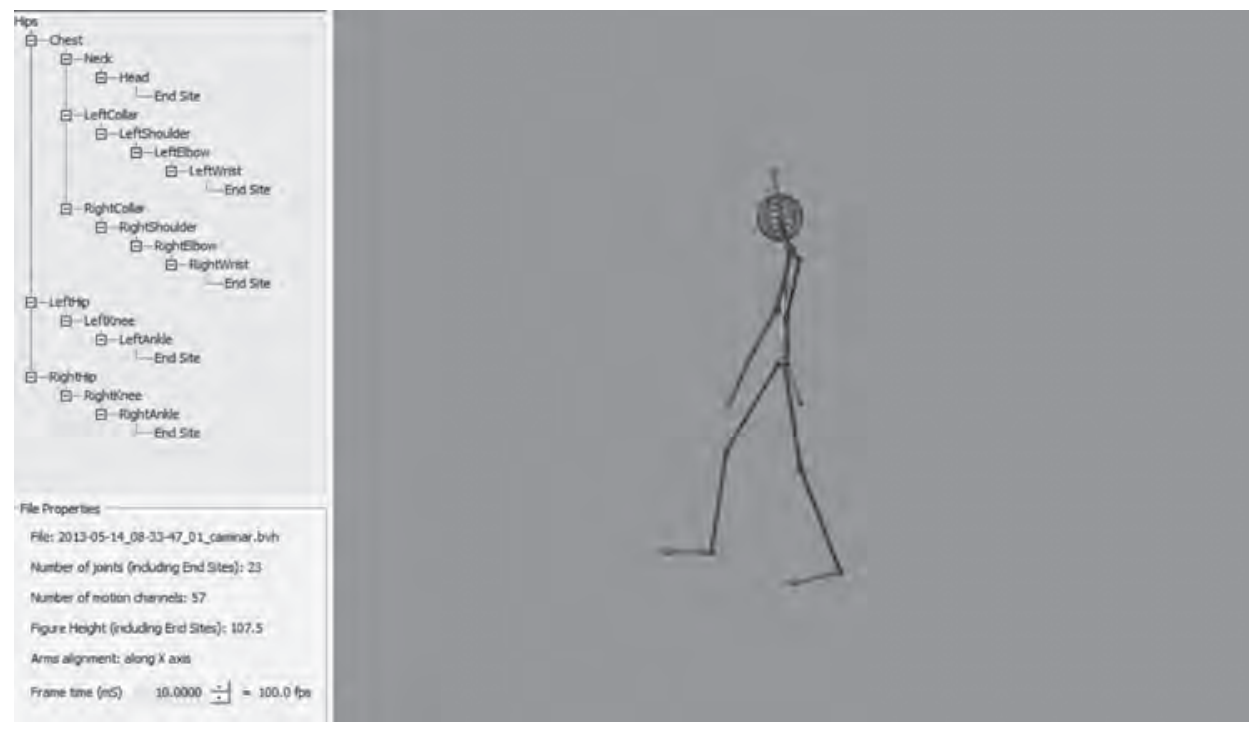

Figura 3. Formato de los archivos guardados.

Gallahue, 1985; Muñoz, 1990; Pandy \& Andriacchi, 2010; Ramón, 2007; Wickstrom, 1990). En particular, se siguieron las secuencias de Wickstrom (1990), para la evaluación del progreso de las formas de andar y de correr y, de $\mathrm{Mc}$ Clenaghan \& Gallahue (1985), las progresiones en el desarrollo de los patrones seleccionados.
Representación y caracterización de secuencias de imágenes: Con el fin de obtener representaciones convenientes de los datos registrados, se disminuyó la carga informativa, mediante el uso de técnicas de reducción de dimensión no lineal, que preserven las relaciones dinámicas de las secuencias de imágenes, teniendo en cuenta la conservación de la 
estructura local y global de los datos, que modelan el fenómeno. En particular, se emplearon métodos como Locally Linear Embedding (Saul \& Roweis, 2003) y Isometric Feature Mapping (Tenenbaum et al. 2000). Además, como resultado de aplicar estas técnicas, se obtuvo un modelo que representa el comportamiento dinámico de un ejercicio físico predeterminado (Bowden, 2000; Belkin \& Niyogi, 2003; Ormoneit et al. 2005). Dicho modelo es explicado por un conjunto de variables y sus relaciones topológicas entre observaciones.

Además, se emplearon técnicas de reconocimiento automático de patrones, tales como: Análisis Discriminante Lineal (Webb, 2002) y Máquinas de Vectores de Soporte (Vapnik, 1995), las cuales, aplican las variables anteriormente determinadas, para identificar y juzgar la correcta ejecución de los PBM (Weinberger \& Saul, 2004).

Validación y pruebas de rendimiento del sistema: Se estudió la efectividad de las técnicas de reconocimiento automatizado para la identificación de las diferencias entre los PBM caminar y correr, y las ejecuciones de los mismos, realizados por la población objetivo. Este análisis, se realizó una vez fueron conocidos las características dominantes de los PBM. La intención era determinar las capacidades del prototipo automatizado, comprobando por medio de pruebas de concordancia, la especificidad y la sensibilidad del sistema, apoyadas en la evaluación realizada por especialistas clínicos y del deporte.

\section{RESULTADOS Y DISCUSIÓN}

Con el objetivo de caracterizar los PBM caminar y correr, en niñas de 5 a 8 años, residentes en área urbana de Manizales, por medio de técnicas de reconocimiento automatizado de patrones, fundamentadas en métodos de extracción no lineal de características, fueron evaluadas 48 niñas seleccionadas al azar simple, con unas medias de 7,05 $\pm 0,99$ años de edad, $116,0 \pm 6,83 \mathrm{~cm}$. de talla y $22,6 \pm 4,13 \mathrm{~kg}$ de masa corporal, pertenecientes a un colegio de carácter privado de Manizales. Las características sociodemográficas aparecen en la tabla 1.

En el PBM caminar, el 29,2\% mostró un patrón maduro. Entre las deficiencias detectadas resaltaron, por su alta frecuencia, problemas en miembros superiores $(50 \%)$, desglosados en: falta de alternancia de brazos $(27,1 \%)$, uno o ambos brazos quietos $(14,5 \%)$, brazos muy extendidos $(2,1 \%)$, asimetría de hombros $(2,1 \%)$, mayor flexión de un codo $(2,1 \%)$ y asimetría de manos $(2,1 \%)$. Este resultado, frente a lo planteado por Sutherland et al. (1980), de que la primera aparición del balanceo en oposición de los brazos fue a los 1,5 años y que sistemáticamente aumentaba con la edad, hasta los 3,5 años y por Burnett \& Johnson (1971) y Sutherland et al. (1980), que el balanceo recíproco de brazos no se observa en infantes al inicio de la marcha independiente, pero se desarrolla durante la infancia, lleva a la conclusión que las niñas evaluadas presentan un retraso en esta característica del patrón evaluado, pues para una edad media de 7,05 años (mínima de 6,3), ya deberían exhibir un patrón maduro.

Un 12,5\% de la muestra evaluada presentó problemas en el tren inferior, desglosado en: inversión de pies (6,3\%), flexión exagerada de rodillas $(2,1 \%)$, longitud del paso desigual $(2,1 \%)$ y movimiento exagerado de la cadera $(2,1 \%)$, datos muy similares a los encontrados por Rodríguez (s.f.), quien registró, como problemas globales, la falta de amplitud de movimientos y mucha rigidez en los movimientos, con $4,2 \%$, cada uno.

Si bien se esperaría que a medida que las niñas crecen, sus PBM mejoran, dada la ganancia en control motor y la acumulación de experiencias, se evidenció que, por el contrario,

Tabla 1. Características sociodemográficas de la muestra.

\begin{tabular}{|c|c|c|c|c|c|}
\hline Características & Talla $(\mathrm{cm})$. & Peso (kg.) & Cant. & $\%$ & $X \pm s$ \\
\hline \multicolumn{6}{|l|}{ Edad (años) } \\
\hline $5-6$ & $107,0 \pm 2,94$ & 17,52 & 4 & 8,3 & $5,84 \pm 0,14$ \\
\hline $6-7$ & $110,0 \pm 4,08$ & 18,64 & 24 & 50 & $6,20 \pm 0,03$ \\
\hline $7-8$ & $118,6 \pm 5,55$ & 22,03 & 11 & 22,9 & $7,38 \pm 0,27$ \\
\hline $8-9$ & $123,6 \pm 4,24$ & 24,51 & 9 & 18,8 & $8,55 \pm 0,33$ \\
\hline Todas & $116,0 \pm 6,83$ & 21,09 & 48 & 100 & $7,05 \pm 0,99$ \\
\hline \multicolumn{6}{|l|}{ Escolaridad (grado) } \\
\hline Transición & & & 23 & & $47,9 \%$ \\
\hline 1 & & & 16 & & $33,3 \%$ \\
\hline 2 & & & 9 & & $18,8 \%$ \\
\hline
\end{tabular}


en cuanto al correr, solamente un 47,9\% presentó el patrón maduro normal, mientras que en el tren superior, se registraron las siguientes deficiencias: falta de flexión de brazos $(14,6 \%)$, no alternancia de brazos $(8,4 \%)$, movimiento desordenado de brazos (4,2\%), asimetría de manos, asimetría en la flexión de brazos, brazo en inversión y brazos quietos $2,1 \%$, cada uno. En miembros inferiores, las deficiencias fueron: inversión de pies $(4,2 \%)$, movimiento asimétrico de pies $(2,1 \%)$, longitud del paso desigual $(2,1 \%)$ y piernas muy abiertas (2,1\%); a nivel pélvico, se presentaron retracción y falta de estabilidad, con $2,1 \%$, cada una. Como problema global, se manifestó desequilibrio, con un 2,1\%.

La distribución de la muestra en términos de normalidad y de anormalidad de los dos patrones evaluados por grupos de edad, se presenta en la tabla 2.
Mc Clenaghan \& Gallahue (1985) sugieren que a los siete años deben estar maduros los patrones evaluados, por lo tanto, se puede concluir, que la maduración de los PBM caminar y correr está atrasada frente a lo esperado para la edad, con un $56,12 \%$, de las niñas, reflejando patrones inmaduros para su edad, deficiencias que deberían estar superadas desde varios años atrás, por lo cual, se recomienda incluir, en el área de educación física, una unidad dedicada a trabajar estos temas, para superar las deficiencias evidenciadas.

Similar a lo encontrado por Rodríguez (s.f.), se observó que las pruebas que más dificultades presentaron fueron la pobreza de automatismo y le siguió, en menor escala, la cabeza y el cuello adelantados, viéndose afectada toda la actividad motriz y, por ende, la vida del niño, ya que transcurre en una combinación de movimiento y de pensamiento. Asimismo,

Tabla 2. Deficiencias en los PBM por grupos de edad (porcentajes).

\begin{tabular}{|c|c|c|c|c|}
\hline $\begin{array}{l}\text { Grupo etario } \\
\text { (años) }\end{array}$ & $\begin{array}{r}\text { Edad } \\
(\mathrm{X} \pm \mathrm{Ds})\end{array}$ & Evaluación & Caminar & Correr \\
\hline \multirow{2}{*}{$\begin{array}{l}\text { Todas } \\
(\mathrm{n}=48)\end{array}$} & \multirow{2}{*}{$7,05 \pm 0,99$} & Normal & $30,61 \%$ & $36,75 \%$ \\
\hline & & Anormal & $57,14 \%$ & $46,44 \%$ \\
\hline \multirow{2}{*}{$\begin{array}{l}5 \\
(\mathrm{n}=4)\end{array}$} & \multirow{2}{*}{$5,84 \pm 0,14$} & Normal & $50,00 \%$ & $66,67 \%$ \\
\hline & & Anormal & $50,00 \%$ & $33,33 \%$ \\
\hline \multirow{2}{*}{$\begin{array}{l}6 \\
(n=24)\end{array}$} & \multirow{2}{*}{$6,20 \pm 0,03$} & Normal & $23,81 \%$ & $36,84 \%$ \\
\hline & & Anormal & $76,19 \%$ & $63,16 \%$ \\
\hline \multirow{2}{*}{$\begin{array}{l}7 \\
(\mathrm{n}=11)\end{array}$} & \multirow{2}{*}{$7,38 \pm 0,27$} & Normal & $44,44 \%$ & $50,00 \%$ \\
\hline & & Anormal & $55,56 \%$ & $50,00 \%$ \\
\hline \multirow{2}{*}{$\begin{array}{l}8 \\
(n=9)\end{array}$} & \multirow{2}{*}{$8,55 \pm 0,30$} & Normal & $37,50 \%$ & $37,50 \%$ \\
\hline & & Anormal & $62,50 \%$ & $62,50 \%$ \\
\hline
\end{tabular}

se encontró similitud con otros resultados, que apoyan una análisis del desarrollo tardío de los patrones fundamentales (Braga et al. 2009; Brauner \& Valentini, 2009; Clark, 2007; Gallahue \& Donnelly, 2008).

En conclusión, sí fue posible identificar, de manera automática, deficiencias en los PBM, mediante técnicas de extracción no lineal de características.

Agradecimientos: Al Colegio Ravasco (estudiantes, profesores, padres de familia y directivos), al Tenis Club de Manizales y a los estudiantes Claudia Lorena Tabarquino y Jorge Bustamante. Financiación: Este estudio fue financiado por las Universidades de Caldas y Nacional sede Manizales. Conflicto de intereses: El manuscrito fue preparado y revisado con la participación de todos los autores, quienes declaramos que no existe ningún conflicto de intereses, que ponga en riesgo la validez de los resultados presentados.

\section{BIBLIOGRAFÍA}

1. ANGLADA, P. 2010. El patrón motor del arrastre: punto de partida. Rev. Int. Med. Cienc. Act. Fís. Dep. (España) 10(39):458-469. Disponible desde Internet en: http://cdeporte.rediris.es/revista/revista39/artpatron174.htm (con acceso 15/01/2014).

2. BAENA, A.; GRANERO, A.; RUIZ, P.J. 2010. Procedures and instruments for the measurement and evaluation of motor development in the educations system. J. Sport Health Res. (E.E.U.U.) 2(2):63-18. 
3. BARABAS, A. s.f.. Biomechanics of sport patterns and motor development. Department of Biomechanics, Hungarian University of Physical Education. 120p.

4. BELKIN, M.; NIYOGI, P. 2003. Laplacian eigenmaps for dimensionality reduction and data representation. Neural Comput. 15:1373-1396.

5. BOWDEN, R. 2000. Learning statistical models of human motion, analysis and synthesis. In IEEE Workshop on Human Modelling. (E.E.U.U.).186p.

6. BRAUNER, L.M.; VALENTINI, N.C. 2009. Análise do desempenho motor de crianças participantes de um programa de atividades físicas. Rev Educ. Física/ UEM, Maringá. 20(2):205-216.

7. BRAGA, R.K.; KREBS, R.J.; VALENTINI, N.C.; TKAC, C.M. 2009. A influência de um programa de intervenção motora no desempenho das habilidades locomotoras de crianças com idade entre 6 e 7 años. Rev. Educ. Física/UEM, Maringá, 20(2):171-181.

8. BURNETT, C.N.; JOHNSON, E.W. 1971. Development of gait in childhood. Part II. Developmental Med. Child Neurol. 13:207-215.

9. CLARK, J.E. 2007. On the problem of motor skill development. J. Phys. Educ. Recreat. Dance. 78(1):58-65.

10. FERRAZ, O.L. 1992. Desenvolvimento do padrão fundamental de movimento correr em crianças: um estudo semilongitudinal. Rev. Paulista Ed. Física. 6(1):26-34.

11. FROST, J.L.; WORTHAM, S.C.; REIFEL, S. 2010. Physical Development and the Acquisition of Motor Skills. Pearson Allyn Bacon Prentice Hall. (E.E.U.U.). 255p.

12. FROST, J.L. 2006. The dissolution of children's outdoor play: Causes and consequences. Conference paper delivered at The Value of Play: A forum on risk, recreation and children's health. (Estados Unidos). Disponible desde Internet en: http://cgood.org/assets/ attachments/Frost_-_Common_Good___FINAL.pdf (con acceso 21/06/2006).

13. GALLAHUE, D.L.; OZMUN, J.C. 2005. Compreendendo o desenvolvimento motor: bebês, crianças, adolescentes e adultos. 3a ed. São Paulo: Phorte. 524p.

14. GALLAHUE, D.L.; DONNELLY, F.C. 2008. Educação Física desenvolvimentista para todas as crianças. $4^{a}$. Ed. São Paulo: Phorte. 725p.
15. GARCIA, C.; GARCIA, L.; FLOYD, J.; LAWSON, J. 2002. Improving public health through early childhood movement programs. J. Phys. Educ. Recreat. Dance. 73(1):73:77.

16. GOODWAY, J.D. 2009. What's skill got to do with it? A developmental approach to promoting physical activity in urban youths. J. Phys. Educ. Recreat. Dance. 80:1-60.

17. GIL-AGUDO, A.M.; PÉREZ-RIZO, E.; DEL ALMA-ESPINOZA, A.; CRESPO-RUIZ, B.; PÉREZ-NOMBELA, S.; SÁNCHEZ-RAMOS, A. 2009. Comparative biomechanical gait analysis of patients with central cord sindrome walking with one crutch and two crutches. Clin. Biomech. 24(7):551-557.

18. GROSSER, M.; HERMANN, H.; TUSKER, F.; ZINTL, F. 1991. El movimiento deportivo. Bases anatómicas y biomecánicas. Ed. Martínez Roca (España). 199p.

19. HAYWOOD, K.M. 1986. Life span motor development. Champaign: Human Kinetics. 327p.

20. HEWES, J. 2006. Let The Children Play: Nature's Answer to Early Learning. Edmonton: Grant MacEwan College. (E.E.U.U.) 177p.

21. KWON, Y.H. 2009. Temáticas actuales en análisis del movimiento y sus aplicaciones en el deporte. Ed. Funámbulos. Universidad de Antioquia (Colombia). 134p.

22. LEDEBT, A. 2000. Changes in arm posture during the early acquisition of walking. Infant Behavior \& Developm. 23(1):79-89.

23. MC CLENAGHAN, B.A.; GALLAHUE, D.L. 1985. Movimientos fundamentales. Su desarrollo y rehabilitación. BsAs: Méd. Panam. (Argentina). 217p.

24. MEINEL, K. 1977. Didáctica del Movimiento. Ensayo de una Teoría del Movimiento en el Deporte desde el Punto de Vista Pedagógico. La Habana (Cuba). 317p.

25. MUÑOZ, L.A. 1990. Desarrollo Motor y Educación Física Infantil. Teoría Básica. Universidad Surcolombiana (Colombia). 278p.

26. MURRAY, M.P.; KORY, R.C.; BERNARD, E.J. 1967. Patterns of sagittal rotation of the upper limbs in walking: a study of normal men during free and fast speed walking. Physical Therapy. 47:272-284. 
27. NITSCH, J.; NEUMAIER, A.; DE MARÉES, H.; MESTER, J. 2002. Entrenamiento de la técnica. Contribuciones para un enfoque interdisciplinario. Ed. Paidotribo (España). 585p.

28. ORMONEIT, D.; SIDENBLADH, H.; BLACK, M.J.; HASTIE, T. 2005. Learning and tracking cyclic human motion. En: Advances in Neural Information Processing Systems - NIPS (Alemania) 14(1): 894-900.

29. PANDY, M.G.; ANDRIACCHI, T. 2010. Muscle and joint function in human locomotion. Ann. Rev. Biomed. Eng. (E.E.U.U.) 12:401-433.

30. RAMÓN, G. 2007. Técnica, Biomecánica y aprendizaje motriz. Ed. Funámbulos, Universidad de Antioquia (Colombia). 134p.

31. RAMOS, S.; MELO, L.G.; ALZATE, D.A. 2007. Evaluación Antropométrica y Motriz Condicional de Niños y Adolescentes. Ed. Universidad de Caldas (Colombia). 146p.

32. RODRÍGUEZ R, J.E. s.f.. Evaluación de la marcha y la postura en niños de edad preescolar en el municipio de Matanzas. Facultad de Cultura Física Universidad de Matanzas "Camilo Cienfuegos". (Cuba). Disponible desde Internet en:

www.bibliociencias.cu/gsdl/collect/libros/index/assoc/...dir/doc (con acceso 14/02/14).

33. RUIZ PÉREZ, L.M. 1987. Desarrollo motor y actividades físicas. Ed. Gymnos. (España). 246 p.

34. SAUL, L.K.; ROWEIS, S.T. 2003. Think globally, fit locally: Unsupervised learning of low dimensional manifolds. Mach. Learn. Res. 4:119-155.

35. SCHEIRMAN, G.L.; HATFIELD, D. 2009. Técnicas de captura óptica para la valoración del desempeño deportivo. En: Biomecánica deportiva y control del entrenamiento. Ed. Funámbulos, Universidad de Antioquia. (Colombia).134p.
36. SEEFELDT, V.; REUSCHIEIN, S.; VOGEL, P. 1972. Sequencing motor skills within the physical education curriculum. Ed. AAPHERD. (EE.UU.). 256p.

37. SILVA, G. 2002. Diccionario Básico del Deporte y la Educación Física. Ed. Kinesis (Colombia). 328p.

38. SUTHERLAND, D.H.; OLSHEN, R; COOPER, I.; WOO, S.L. 1980. The development of mature gait. J. Bone Joint Surg. Am. (E.E.U.U.) 62:336-353.

39. THELEN, E.; SMITH, L.B. 1994. A dynamic systems approach to the development of cognition and action. Cambridge: MIT Press. 377p.

40. TENENBAUM, J.B.; DE SILVA, V.; LANGFORD, J.C. 2000. A global geometric framework for nonlinear dimensionality reduction. Science. 290:2319-2322.

41. ULRICH, D. 2000. The test of Gross motor development. 2 ed. Austin: Prod- Ed. C.D.

42. VAPNIK, V. 1995. The Nature of Statistical Learning Theory. Ed. Springer. (EE.UU.) 314p.

43. WEBB, A.R. 2002. Statistical Pattern Recognition. John Wiley and Sons Ltd. (EE.UU.) 913p.

44. WEINBERGER, K.Q.; SAUL, L.K. 2004. Unsupervised learning of image manifolds by semidefinite programming. In: Proceedings of the IEEE Conference on Computer Vision and Pattern Recognition (CVPR04). (Estados Unidos). 1643p.

45. WICKSTROM, R.L. 1990. Patrones motores básicos. Ed. Alianza (España). 293p.

Recibido: Febrero 19 de 2014

Aceptado: Octubre 23 de 2014

\section{Como citar:}

Ramos, S.; Vidarte, J.A.; Gómez, L.A. 2014. Patrones básicos caminar y correr en niñas de 5 a 8 años de edad mediante extracción no lineal de características. Rev. U.D.C.A Act. \& Div. Cient. 17(2): 351-359. 